bilaterally (figure, B and C). Ten months later, MRI disclosed atrophy of the putamen and caudate nuclei-instead of the high signal intensity seen previously - and enlargement of the anterior horns of the lateral ventricles (figure, D). After admission to our hospital, he was treated with levodopa/carbidopa, amantadine hydrochloride, dihydroxyphenyl serine, bromocriptine, or pergolide mesilate and given physiotherapy. The drugs yielded no improvement. His clinical course was progressive and paralleled the MRI changes in the striatum.

Central pontine myelinolysis usually manifests as disturbance of consciousness, pseudobulbar palsy, tetraplegia, and positive pyramidal signs. Extrapontine myelinolysis occurs in about $10 \%$ of patients with central pontine myelinolysis and often affects the basal ganglia, mainly the caudate and putamen. ${ }^{1}$

This patient showed no signs or symptoms of central pontine myelinolysis, and MRI disclosed no evidence for it. We think that the parkinsonism seen in our patient is a clinical manifestation of extrapontine myelinolysis that occurred after rapid correction of hyponatraemia, for the following reasons. Firstly, typical central pontine myelinolysis is not associated with parkinsonism. Secondly, basal ganglia abnormalites on MRI, high signal intensities on T2 weighted images in the early stage, and atrophy of putamen and caudate nuclei in the chronic stage, are correlated with gradual worsening of his parkinsonism.

Among the 18 reported cases of central pontine myelinolysis/extrapontine myelinolyis associated with parkinsonism and involuntary movements (dystonia and chorea) parkinsonism was mentioned in eight. All except one patient responded to antiparkinsonian drugs and showed very good prognosis despite acute or delayed onset of parkinsonism. ${ }^{2}$ Dystonia was reported in nine cases. By contrast with the parkinsonism, all patients except one showed delayed onset and in five of them it persisted.Okeda et $a l^{3}$ and Morikawa et $a l^{4}$ described patients with extrapontine myelinolysis without central pontine myelinolysis who showed no signs of parkinsonism or dystonia. One patient described by Morikawa et $\mathrm{al}^{4}$ showed only extrapontine myelinolysis on MRI although both central pontine myelinolysis and extrapontine myelinolysis were confirmed at postmortem. The patient reported by Niwa $e t a^{5}$ is the only one who showed dystonia and rigidity, and had extrapontine myelinolysis without central pontine myelinolysis on MRI. Compared with the previously reported patients, our patient is unusual for two reasons. Firstly, the clinical characteristics of acute parkinsonism with out response to antiparkinsonian drugs, its progressive course, and mild dystonia in the fingers, not associated with a pontine syndrome. Secondly, the progressive parkinsonism correlated with extrapontine myelinolysis shown on MRI, the increased signal intensity on T2 weighted images in the striatum in the acute stage, and decreased signal intensity on $\mathrm{T} 2$ weighted images and atrophy of the striatum in the chronic stage.

Brain MRI most often discloses a central pontine myelinolysis/extrapontine myelinolysis lesion as an area of persisting, prolonged $\mathrm{T} 1$ and $\mathrm{T} 2$ relaxation times within the basis pons and striatum. Absence of imaging changes on MRI does not necessarily indicate the absence of associated disease, and it remains possible that pathological changes had taken place in the pons, which were not detected on imaging.

$$
\begin{array}{r}
\text { ITSUROU TOMITA } \\
\text { HIDEYO SATOH } \\
\text { AKIRA SATOH } \\
\text { MAKIKO SETO } \\
\text { MITSUHIRO TSUIIHATA } \\
\text { Neurology Section, Nagasaki-kita Hospital, } \\
\text { Nagasaki 85,2, Japan } \\
\text { TOSHIRO YOSHIMURA } \\
\text { Department of Occupational Therapy, } \\
\text { School of Allied Medical Sciences, } \\
\text { Nagasaki University, Nagasaki 852, fapan }
\end{array}
$$

Correspondence to: Dr I Tomita, Neurology chome, Nagasaki 852, Japan.

1 Wright DG, Laureno R, Victor M. Pontine and extrapontine myelinolysis. Brain 1979; 102:361-85.

2 Maraganore DM, Folger WN, Swanson JW, Ahlskog JE. Movement disorders as sequelae of central pontine myelinolysis: rep
three cases. Mov Disord 1992;7:142-8.

3 Okeda R, Kitano M, Sawabe M, Yamada I, Yamada $\mathbf{M}$. Distribution of demyelinating lesions in pontine and extrapontine myelinolysis - three autopsy cases including one case devoid of central pontine myelinolysis. Acta Neuropathol (Berl) 1986;69:259-66.

4 Morikawa F, Tashiro K, Maruo Y, Nomura M, Hamada K, Kashiwaba T. MR imagin of pontine and extrapontine myelinolysis. $f$ Comput Assist Tomogr 1988;12:446-9.

5 Niwa $K$, Matsushima $K$, Yamamoto $M$, Shinohara Y. A case of extrapontine myelinolysis demonstrated on MRI. Clin Neurol (Tokyo) 1995;31:327-30.

\section{Prospective study of neurorehabilitation in multiple sclerosis}

The importance of the role of structured programmes of neurorehabilitation in specialist units is becoming increasingly recognised. ${ }^{1}$ It is known that such rehabilitation programmes confer benefit in the management of stroke ${ }^{2}$; much less is known of their effect on progressive disorders such as multiple sclerosis. In a previous study of 79 patients with multiple sclerosis a significant improvement in disability score (the Barthel index) occurred after a short structured rehabilitation programme; only 14 had improved neurologically during the period of rehabilitation, suggesting a beneficial effect on those with stable or deteriorating function. ${ }^{3}$ One important question is whether or not this improvement in function is maintained after discharge into the community.

Consecutive patients with clinically definite multiple sclerosis admitted after a multidisciplinary assessment to the neurorehabilitation unit of the National Hospital for Neurology and Neurosurgery were studied. All assessments were carried out by a single observer (DK) within 24 hours of admission and discharge. Each patient underwent a full neurological examination from which the expanded disability scale score (EDSS) ${ }^{4}$ was obtained. Disability and handicap were scored using the functional independence measure (FIM, range 13 to 90 , in which an increase in score reflects an improvement in disability $)^{5}$ and the environmental status scale ${ }^{6}$ (ESS, range 0 to 35 , in which a decrease in score reflects a reduc- tion in handicap) which was based on patient reports to direct questioning about each functional task.

Patients underwent an individual, goal oriented rehabilitation programme based on an admission assessment by a multidisciplinary team composed of therapists, nurses, and a psychologist.

Three months after discharge all patients were invited to return to an outpatient clinic where the assessments were repeated by the same observer. Paired observations and group data comparisons were made using the Wilcoxon matched pairs signed rank sum test and the Mann-Whitney $U$ test respectively.

Forty seven patients were studied (75\% female) with a mean (SD) age of 40 (11) years and median (range) duration of disease of 13 (three to 28 ) years. Thirty nine were progressive (two were primary progressive), and eight had a relapsing-remitting course. Nineteen had had a relapse before admission, including all relapsing-remitting patients. The median (range) hospital stay was 15 ( 7 to 87 ) days. The table shows the median (range) EDSS, FIM, and ESS scores on admission and discharge. Eight patients $(17 \%)$ improved on the EDSS, median change was 0 (range 0 to 2). There was improvement in $41(87 \%)$ patients on the FIM, median change 6 (range 68 to -1 ) $(\mathrm{P}<0.001)$. Twenty two $(47 \%)$ improved on the ESS, median change 0 (range -18 to +12).

Forty four patients were reviewed three months after discharge. The table shows the median (range) EDSS, FIM, and ESS on follow up. A deterioration in FIM occurred in six $(14 \%)$ patients whereas $13(30 \%)$ had improved on the ESS. Eleven patients had new symptoms (eight had had a relapse, three had undergone progression). In only two cases, however, was this change sufficient to cause a change on the EDSS. The median change in FIM was -3 ( 1 to -33 ) in those with new symptoms and 0 (14 to 27 ) in those who had remained stable $(P<0.02)$, and there was no difference between median change in the two groups in EDSS and ESS (median 0, range $-2 \cdot 0$ to 0 and 0 to $2 \cdot 5$, and 0 to 0 and -4 to 0 respectively) $(P>0.5)$.

In this study of consecutive patients selected for a period of intensive neurorehabilitation a significant improvement in function was made in most $(87 \%)$ patients. In addition, the present study considered the crucial question of whether or not this improvement may be carried over after discharge. Functional gains were maintained in $86 \%$ of the 44 patients followed up at three months, $75 \%$ of whom had been clinically stable.

These findings provide further insights into the role which neurorehabilitation has in the management of multiple sclerosis. Handicap tended to improve further after discharge, reflecting work planned during the rehabilitation programme being carried out in the home, such as the provision of hand rails and ramps and the introduction of

Median (range) scores on admission, discharge, and at three month follow up in all patients studied ( $n=47$ for admission and discharge scores, and 44 for follow up scores)

\begin{tabular}{lccc}
\hline & Admission & Discharge & $\begin{array}{c}\text { Three month } \\
\text { follow up }\end{array}$ \\
\hline EDSS & $7 \cdot 5(5 \cdot 0-9 \cdot 0)$ & $7 \cdot 0(4 \cdot 0-9 \cdot 0)$ & $7 \cdot 0(2 \cdot 5-9 \cdot 0)$ \\
FIM (motor) & $66(13-85)$ & $80(18-90)$ & $77(18-90)$ \\
ESS & $19(2-31)$ & $18 \cdot 5(4-30)$ & $17(3-32)$ \\
\hline
\end{tabular}


community services. There is clearly a need for the development of a randomised controlled trial to confirm these results; the difficulties involved in undertaking such a study would be great, although preliminary results from a recent study suggest that they are not insurmountable. ${ }^{8}$

D KIDD

Neurorehabilitation section, University Department of Clinical Neurology, Institute of Neurology, Queen
Square, London WCIN 3BG, UK

Correspondence to: $\mathrm{Dr}$ AJ Thompson, The surgery, Queen Square, London WC1N 3BG UK.

1 Ward C, McIntosh S. The rehabilitation process: a neurological perspective. In process: a neurological perspective. In Greenwood R, Barnes MP, MeMillan TM, Ward CD, eds. Neurological rehabilitation. London: Churchill Livingstone, 1993:13-27.

2 Indredavik B, Bakke F, Solberg R, Rokseth R Hacheim LL, Holme I. Benefit of a stroke unit: a randomised controlled trial. Stroke 1991;22:1026-31.

3 Kidd D, Howard RS, Losseff NA, Thompson AJ. The benefit of neurorehabilitation in MS Clinical Rehabilitation 1995;9:198-203. 4 Kurtzke JF. Rating neurologic impairment in
MS: an expanded disability status scale (EDSS). Neurology 1983;37:1444-52.

5 Hamilton BB, Granger CV, Sherwin FS, Zielezny M, Tashman JS. A uniform national data system for medical research. In: Fuhrer $\mathrm{data}$ system for medical research. In: Fuhrer $\mathrm{M}$, ed. Rehabilitation outcome: analysis and
measurement. Baltimore: Paul $\mathrm{H}$ Brooks, measurement.

6 A symposium on the minimal record of disability. Acta Neurol Scand 1984;101(suppl): 169-207.

7 Johnson J, Thompson AJ. Rehabilitation in a neurosciences centre: the role of exper assessment and selection. British fournal of Therapy and Rehabilitation 1996;3:303-8.

8 Freeman JA, Langdon DW, Hobart JC, Thompson AJ. The impact of rehabilitation on disability and handicap in progressive MS: a randomised controlled trial. $¥$ Neurol 1996;243(suppl 2):S17.

Eye opening reflex triggered by flexion of an arm: a manifestation of decerebrate response in diffuse bilateral hemispheric damage

Some comatose patients with acute and severe brain damage recover from a continuous sleep-like stage and enter a state in which they show periodic wakefulness when the eyes stay open and move, but never show a recognisable mental function. This syndrome is known as the permanent vegetative state, in which responses are limited to primitive postural and reflex movements. ${ }^{1}$ In two such patients we noted a peculiar but constant bilateral eye opening reflex triggered by a particular stimulus - namely, flexion of the arm at the elbow joint.

A 47 year old man had a cardiac arrest due to generalised status epilepticus. After successful resuscitation, he fell into a persistent coma. The pupils were dilated and not reactive to light. All limbs were flaccid. An immediate brain CT showed no abnormality. A few days later, the patient started to show intermittent decerebrate posturing to noxious stirnuli, such as intratracheal intubation and transoral suction. Two weeks later, a sleep-wakefulness cycle returned, and spontaneous breathing was resumed.

When awake, his eyes were wide open, and moved from side to side spontaneously. Spontaneous eye blinking was present, but he never followed objects with his eyes, or responded to visual threat or verbal commands. The pupils were not dilated, and were reactive to light. The corneal reflexes were present on both sides. There were occasional spontaneous mastications, swallowing, and yawning, but no voluntary movements of the limbs. The tendon reflexes were brisk, and the plantar response was extensor on the left side. A follow up CT showed ischaemic infarction in bilateral striate areas and severe diffuse hemispheric atrophy. A scalp EEG was isoelectric.

In the sleep stage, his eyes remained closed. However, whenever an examiner briskly flexed either of the patient's arms at the elbow, the patient's eyes opened wide and full without grimace and pupillary dilatation (figure). Once opened, they remained so for a few seconds, suggesting their tonic nature. Further, with the opening of the eyes, the head retracted and the limbs on the opposite side extended rigidly. His jaw was closed. Subsequently, spontaneous blinking, head shaking, and occasionally yawning occurred in that sequence, and he entered a waking state. The other stimuli, such as pinching of the skin around the elbow or strong compression of the brachia muscles and fingertips, did not induce either the eye opening or extension of the head and limbs. Also, when the examiner lifted the upper lids manually and released them, both eyes drooped promptly. This unique eye opening response was a constant phenomenon and we confirmed the presence of the response every day until the patient died of heart failure three months later.

A 60 year old man with a history of callosal infarction developed a cardiac arres after suffocation, from which he was resuscitated but he did not recover consciousness. His pupils were dilated, and sluggishly reactive to light. All the limbs were flaccid. Brain CT on the third day in hospital showed dif fuse bilateral low density areas with oedema and enlargement of the lateral and third ven tricles. A scalp EEG on the 20th day in hospital was isoelectric. Auditory evoked responses taken on the same day indicated the presence of electrical activity at the midbrain level but not higher.

On that day, sleep-wakefulness cycles were seen. No response to verbal commands was elicited. Respiration was spontaneous via an intratracheal airway. When awake, his eyes were wide open, with spontaneous blinking and horizontal roving eye movements. Oculocephalic reflexes were present. There was spontaneous mastication, but no discernible active movements in the limbs.

In sleep, his eyes remained closed and the limbs did not move at all, even if the elbow was pinched strongly or the brachial muscles and fingertips were compressed strongly. When the upper eyelids were lifted manually and released, they drooped immediately. However, when either of the arms was flexed at the elbow passively, the eyes opened wide and full after a brief latency. The pupils did not become dilated and no grimacing occurred. On flexion of the right arm, the upper eyelids elevated tonically with turning of the head to the contralateral left side and rigid extension of the limbs on that side. On flexion of the left arm, the right limbs extended with retraction of the head. The eye opening was sustained for a while, and was followed shortly afterwards by spontaneous blinking and roving eye movements. The patient died of heart failure about six months after the onset.

A reflex eye opening is known as Doll's eye movement. This reflex in comatose patients was probably first described by Cogan, who wrote that opening of the lids is induced by raising of the head or turning of the head from side to side. ${ }^{2}$ This interesting phenomenon was further confirmed by Fisher who wrote that Doll's eyelids designates a remarkable reflex opening of the eyelids when in the comatose patient, the neck is briskly flexed. ${ }^{3}$ Cogan was cautious and stated that whether it is attributable to afferent impulses arising in the neck muscles or in the vestibular end organs has yet to be determined.

The reflex eye opening seen in our two patients does not belong to the above phenomenon. We think that this is a new observation not recorded so far. Both patients had severe hypoxic-ischaemic encephalopathy which was caused by cardiac arrest. In the sleep stage when the patient's eyes were closed, respiration was regular and no movement was seen, a passive brisk flexion of the arm elicited the tonic opening of both eyes not accompanied by pupillary change but by contralateral extension of the limbs. We suggest calling this the "arm triggered eye opening reflex" (or arm EOR).

Eye opening is realised principally by the levator palpebrae muscles via the oculomotor nerve and to a certain degree by Müller's muscle via sympathetic excitation. Because sympathetically innervated smooth Müller's muscle plays only a limited part in eye opening, and as the elevation of the upper eyelid in the two cases was full and was accompa-
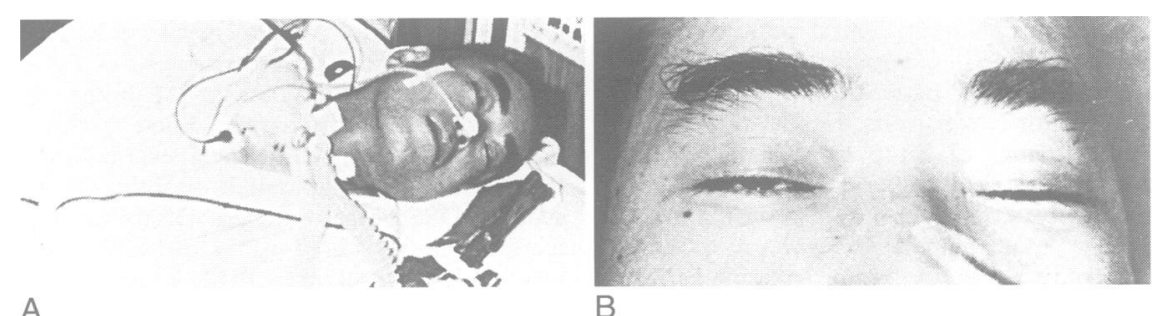

B

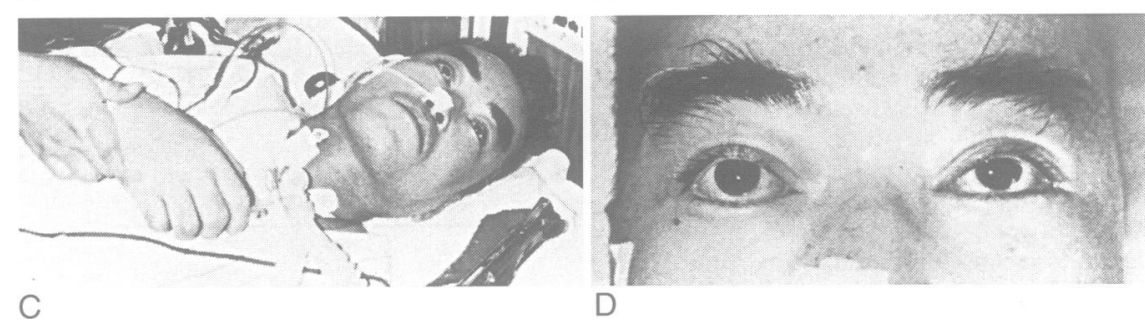

Figure $(A, B)$ In sleep, the eyes were continuously closed. $(C, D)$ Both eyes opened fully when the right arm was put to a flexed posture. 